\title{
Determination of Cerium in Filtrates After Sorption by Stripping Voltammetry on Solid Electrodes
}

\author{
Dmitry V. Zimonin a , Galina V. Burmakina ${ }^{* a, b}$, \\ Tatyana S. Nedelina ${ }^{b}$, Tatyana A. Vereshchagina ${ }^{a}$, \\ Yana Yu. Chernykh and Anatoly I. Rubaylo ${ }^{\mathbf{a}, \mathbf{b}}$ \\ anstitute of Chemistry and Chemical Technology SB RAS \\ FRC "Krasnoyarsk Science Center SB RAS" \\ 50/24 Akademgorodok, Krasnoyarsk, 660036, Russia \\ ${ }^{b}$ Siberian Federal University \\ 79 Svobodny, Krasnoyarsk, 660041, Russia
}

Received 12.10.2017, received in revised form 31.10.2017, accepted 28.11.2017

A stripping voltammetry method was developed for the determination of cerium (III) on graphite electrode as a poorly soluble compound in filtrate after sorption. The cerium (III) concentration presents a good linear relationship over the range of $0.5-10 \mathrm{mg} / \mathrm{l}$. Its linear equation is $I=(0.56 \pm 0.03) \mathrm{c}+$ $+(0.54 \pm 0.10)$, with a correlation coefficient $>0.99$. The detection limit of cerium (III) is $0.1 \mathrm{mg} / \mathrm{l}$. The absence of a significant systematic error is shown by the added-found method. Using the developed technique, the concentration of cerium (III) in the filtrates after sorption has been determined.

Keywords: stripping voltammetry, cerium, sorption filtrate, graphite electrode.

DOI: $10.17516 / 1998-2836-0052$.

(C) Siberian Federal University. All rights reserved

* Corresponding author E-mail address: zimonind89@mail.ru,bgvicct@gmail.com 


\title{
Определение церия в фильтратах после его сорбции методом инверсионной вольтамперометрии \\ на твердых электродах
}

\author{
Д.В. Зимонин ${ }^{\mathrm{a}}$, Г.В. Бурмакина ${ }^{\mathrm{a}, \boldsymbol{\sigma}}$, Т.С. Неделина \\ Т.А. Верещагина ${ }^{a}$, Я.Ю. Черных ${ }^{a}$, А.И. Рубайло ${ }^{a, 0}$ \\ ${ }^{a}$ Институт химии и химической технологии СО РАН \\ ФИЦ «Красноярский научный иентр СО РАН» \\ Россия, 660036, Красноярск, Академгородок,50/24 \\ ${ }^{6}$ Сибирский федеральный университет \\ Россия, 660041, Красноярск, пр. Свободный, 79
}

Разработана методика определения церия (III) в водных растворах методом инверсионной вольтамперометрии на графитовом электроде в виде малорастворимого соединения. Зависимость аналитического сигнала иерия (III) от его конщентрации в растворе линейна в диапазоне 0.5-10 мг/л и описывается уравнением $I=(0.56 \pm 0.03) c+(0.54 \pm 0.10)$ [коэффициент корреляиии > 0.99]. Предел обнаружения иерия (III) составляет 0.1 мг/л. Методом «введено-найдено» показано отсутствие значимой систематической погрешности. С использованием разработанной методики определено содержание иерия 6 фильтратах после его сорбции.

Ключевые слова: инверсионная вольтамперометрия, церий, фильтраты сорбиии, графитовый электрод.

\section{Введение}

Возрастающий интерес к научным и технологическим исследованиям редкоземельных металлов (РЗМ) обусловлен расширением области их применения в химической технологии и металлургии, что связано с получением всех редкоземельных металлов в чистом виде (99.9 \%) методами ионного обмена, металлотермии и вакуумной дистилляции [1]. Изучение индивидуальных РЗМ, в том числе и церия, позволило выявить у них свойства, необходимые для успешной работы новейших физических приборов (лазеры, термоэлектрические преобразователи, атомные батареи, переносные источники излучений и др.), ядерных реакторов, а также в медицине (изотопы при лечении опухолей). Церий используется в качестве легирующей добавки в сплавах хрома и никеля, сплавы церия с магнием и алюминием - в авиастроении, диоксид церия - для шлифовки и полировки оптического стекла и в качестве катализаторов при получении органических соединений [1-14]. Поэтому необходимо определять церий в различных объектах.

В настоящее время церий определяют различными физико-химическими методами: гравиметрическим, спектрофотометрическим, эмиссионным, вольтамперометрическим, радиохимическим, флюоресцентным, рентгеноспектральным [15-27]. Однако, вследствие близости 
физических и химических свойств РЗМ, определение церия затруднено. Следовательно, разработка новых методик определения церия, в том числе и методами вольтамперометрии, является актуальной. Одним из вольтамперометрических методов, позволяющих определять низкие концентрации вещества, является инверсионная вольтамперометрия (ИВА). Чаще всего в качестве рабочих электродов в ИВА используют ртутьсодержащие электроды [28], что требует их замены на экологически безвредные.

Цель работы - разработка методики определения ионов церия (III) в водных растворах и определение его содержания в фильтратах после сорбции методом инверсионной вольтамперометрии на твердых нетоксичных электродах.

\section{Экспериментальная часть}

Растворы готовили из реактивов не ниже ч.д.а. с применением деионизованной воды, полученной при помощи системы очистки воды Direct-Q 3 (Millipore, France), с электропроводностью менее $10^{-7} \mathrm{OM}^{-1} \mathrm{~cm}^{-1}$. В качестве фоновых электролитов использовали $0.04 \mathrm{M}$ раствор $\mathrm{H}_{3} \mathrm{PO}_{4}, 0.1 \mathrm{M}$ ацетатный буферный раствор, $0.5 \mathrm{M}$ раствор $\mathrm{KIO}_{3}$ и $0.04 \mathrm{M} \mathrm{HCl}+0.2 \mathrm{M} \mathrm{CH}_{3} \mathrm{COOH}$. Растворы церия для последующей сорбции и модельных образцов готовили из $\mathrm{Ce}\left(\mathrm{NO}_{3}\right)_{3}$. $4.5 \mathrm{H}_{2} \mathrm{O}$ (ч.д.а). В качестве сорбента использовали гранулы магнитной композиции ферросфер с

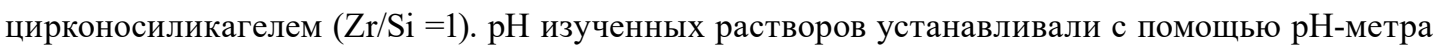
Radelkis (Budapest).

Вольтамперометрические измерения проводили на потенциостате IPC-PRO M с компьютерным и программным обеспечением по трехэлектродной схеме. В качестве рабочих электродов брали платиновый $(\mathrm{Pt})$, стеклоуглеродный $(\mathrm{CV})$ или графитовый $(\Gamma)$ электроды диаметрами 1, 5 и 6 мм соответственно. Вспомогательным электродом служила платиновая проволока, помещенная в стеклянную трубку с пористым фильтром, электродом сравнения насыщенный каломельный электрод (нас.к.э.), соединенный с ячейкой электролитическим мостом, заполненным фоновым электролитом, через капилляр Лунгина. Мешающее влияние кислорода устраняли барботированием очищенного аргона через раствор пробы в течение 15-20 мин.

Для определения церия в фильтратах после его сорбции в электрохимическую ячейку помещали 9 мл раствора фонового электролита $0.04 \mathrm{M} \mathrm{HCl}+0.2 \mathrm{M} \mathrm{CH}_{3} \mathrm{COOH}$, устанавливали $\mathrm{pH}=5.4$, добавляли 1 мл раствора пробы. Концентрирование ионов церия (III) проводили при $\mathrm{E}_{\text {нак }}=1.2$ В. Волну восстановления церия (IV) на вольтамперограммах регистрировали при $\mathrm{E}_{1 / 2}=0.35 \mathrm{~B}$.

\section{Результаты и обсуждение}

Выбор и оптимизация методик вольтамперометрического определения церия в водных растворах на твердых электродах. На основании литературных данных выбраны условия определения ионов церия (III) в водных растворах методом инверсионной вольтамперометрии в виде малорастворимого соединения на твердых электродах. Полученные результаты суммированы в табл. 1.

С использованием этих данных опробован ряд методик определения церия методом ИВА на платиновом, стеклоуглеродном и графитовом электродах в виде малорастворимого

$$
-597-
$$


соединения. Для четырех систем ионов церия (III) с применением ряда фоновых электролитов: $0.04 \mathrm{M} \mathrm{H}_{3} \mathrm{PO}_{4}, 0.1 \mathrm{M} \mathrm{CH}_{3} \mathrm{COOH}+0.1 \mathrm{M} \mathrm{CH}_{3} \mathrm{COONa}, 0.5 \mathrm{M} \mathrm{KIO}_{3}, 0.04 \mathrm{M} \mathrm{HCl}+0.20 \mathrm{M}$ $\mathrm{CH}_{3} \mathrm{COOH}$, и вышеперечисленных рабочих электродов последовательно регистрировали циклические вольтамперограммы фонового электролита и фоновых электролитов с ионами церия (III).

Установлено, что церий (III) электроактивен практически во всех изученных растворах фоновых электролитов в исследуемой области потенциалов. Значения потенциалов полуволн соединений церия (III) приведены в табл. 2.

Однако, как видно из данных табл. 2, ионы церия (III) не образуют электроактивные соединения в водных растворах фонового электролита $0.04 \mathrm{M} \mathrm{H}_{3} \mathrm{PO}_{4}$. Вероятно, это связано с образованием на рабочих электродах малорастворимых фосфатных соединений церия, значения потенциалов полуволн которых находятся вне изученной области потенциалов.

Для дальнейших исследований выбран пик восстановления малорастворимого соединения церия (IV) на графитовом электроде с использованием в качестве фонового электролита $0.04 \mathrm{M} \mathrm{HCl}+0.20 \mathrm{M} \mathrm{CH}_{3} \mathrm{COOH}$ (рис. 1), поскольку он лучше воспроизводится. В этих экспериментальных условиях протекает две электродные реакции: в случае накопления (реакция окисления) $\mathrm{Ce}^{3+}+4 \mathrm{OH}^{-} \rightarrow \mathrm{Ce}(\mathrm{OH})_{4} \downarrow+\mathrm{e}^{-}$, при растворении малорастворимого соединения (реакция восстановления) $\mathrm{Ce}(\mathrm{OH})_{4} \downarrow+\mathrm{e}^{-} \rightarrow \mathrm{Ce}^{3+}+4 \mathrm{OH}^{-}$.

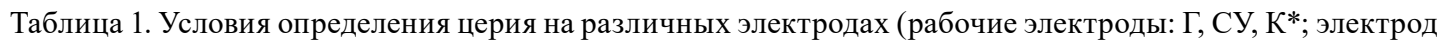
сравнения - нас.к.э.)

Table 1. Conditions for the determination of cerium at various electrodes (working electrodes: G, GC, K*, V vs SCE)

\begin{tabular}{|l|c|c|c|c|c|}
\hline \multicolumn{1}{|c|}{ Фоновый электролит } & $\mathrm{pH}$ & $\begin{array}{c}\text { Потенциал } \\
\text { накопления, В }\end{array}$ & $\begin{array}{c}\text { Потенциал } \\
\text { определения, В }\end{array}$ & $\begin{array}{c}\text { Интервал } \\
\text { определения, M }\end{array}$ & Литература \\
\hline $0.04 \mathrm{M} \mathrm{H}_{3} \mathrm{PO}_{4}$ & 2.5 & +1.0 & $+(0.2-0.8)$ & $10^{-3}-10^{-7}$ & {$[29],[30],[31]$} \\
\hline $\begin{array}{l}0.1 \mathrm{M} \mathrm{CH}_{3} \mathrm{COOH}+ \\
+0.1 \mathrm{M} \mathrm{CH}_{3} \mathrm{COONa}\end{array}$ & 4.6 & $(+0.95 ;+1.05)$ & $+(0.2-0.8)$ & $10^{-3}-10^{-6}$ & {$[30],[32]$} \\
\hline $0.5 \mathrm{M} \mathrm{KIO}_{3}$ & 3.0 & +1.15 & $+(0.2-0.8)$ & $10^{-3}-10^{-7}$ & {$[30],[33]$,} \\
\hline \begin{tabular}{l}
$0.04 \mathrm{M} \mathrm{HCl}_{+}+0.20 \mathrm{M} \mathrm{CH}_{3} \mathrm{COOH}$ \\
\hline
\end{tabular} & 5.2 & +1.1 & $+(0.2-0.8)$ & $10^{-2}-10^{-6}$ & {$[34],[35]$} \\
\hline
\end{tabular}

Примечание: *Г - графитовый, СУ - стеклоуглеродный, К - композиционный.

Таблица 2. Значения потенциалов полуволн ионов церия (III) $(\mathrm{C}=7.5$ мг/л, отн. нас.к.э., v $=25$ мB/c)

Table 2. Values of cerium (III) half-wave potentials $\left(\mathrm{C}=7.5 \mathrm{mg} \mathrm{l}^{-1}, \mathrm{~V}\right.$ vs SCE, scan rate $\left.25 \mathrm{mV} \mathrm{s}^{-1}\right)$

\begin{tabular}{|l|c|c|c|}
\hline \multirow{2}{*}{ Фоновый электролит } & \multicolumn{2}{c|}{$\mathrm{E}_{1 / 2}, \mathrm{~B}$} & $\Gamma^{*}$ \\
\cline { 2 - 4 } & $\mathrm{Pt}^{*}$ & - & - \\
\hline $0.04 \mathrm{M} \mathrm{H}_{3} \mathrm{PO}_{4}$ & - & 0.47 & 0.33 \\
\hline $0.1 \mathrm{M} \mathrm{CH}_{3} \mathrm{COOH}+0.1 \mathrm{M} \mathrm{CH}_{3} \mathrm{COONa}$ & - & 0.18 & - \\
\hline $0.5 \mathrm{M} \mathrm{KIO}_{3}$ & 0.31 & 0.25 & 0.35 \\
\hline $0.04 \mathrm{M} \mathrm{HCl}+0.20 \mathrm{M} \mathrm{CH}_{3} \mathrm{COOH}$ & 0.18 & - & \\
\hline
\end{tabular}

Примечание: *Pt - платиновый, СУ - стеклоуглеродный, Г - графитовый электроды 
Высоту волны, пропорциональную концентрации ионов церия (III), использовали в качестве аналитического сигнала (АС). Для оптимизации АС ионов церия (III) изучено влияние $\mathrm{pH}$ (рис. 2a), времени накопления (рис. 26) и потенциала накопления (рис. 2в) на его характеристики. Варьированием этих трех факторов установлены оптимальные условия накопления и определения ионов церия (III) на графитовом электроде с использованием $0.04 \mathrm{M} \mathrm{HCl}+0.20 \mathrm{M}$ $\mathrm{CH}_{3} \mathrm{COOH}$ в качестве фонового электролита: $\mathrm{pH}=5.4$; концентрирование при $\mathrm{E}_{\text {нак }}=1.2 \mathrm{~B}$ в течение 420 с, регистрация пика при $\mathrm{E}_{1 / 2}=0.35 \mathrm{~B}$.

Оценены метрологические характеристики методики [36]. Найдено, что предел обнаружения ионов церия (III) составляет 0.1 мг/л. Зависимость аналитического сигнала ионов церия (III) линейна от концентрации (с коэффициентом корреляции $>0.99$ ) в диапазоне концентраций ионов церия (III) $0.5-10$ мг/л и описывается уравнением I $=(0.56 \pm 0.03) c+(0.54 \pm 0.10)$. Относительные стандартные отклонения значений потенциалов полуволн в серии параллельных измерений не превышают 0.02 .

Определение церия в фильтратах после его сорбции. С использованием разработанной методики инверсионно-вольтамперометрического определения проанализирован ряд образцов фильтратов церия после его сорбции. Для каждого образца фильтрата снимали холостой опыт. Концентрацию ионов церия (III) в пробах фильтратов устанавливали по градуировочной зависимости. Результаты экспериментов приведены в табл. 3.

Для проверки правильности результатов использовали метод «введено-найдено» (табл. 3), который показал отсутствие значимой систематической погрешности.

Апробирование методики инверсионно-вольтамперометрического определения ионов церия (III) на реальных объектах - фильтратах после его сорбции, показало, что она может быть использована для определения церия (III) в различных водных растворах.

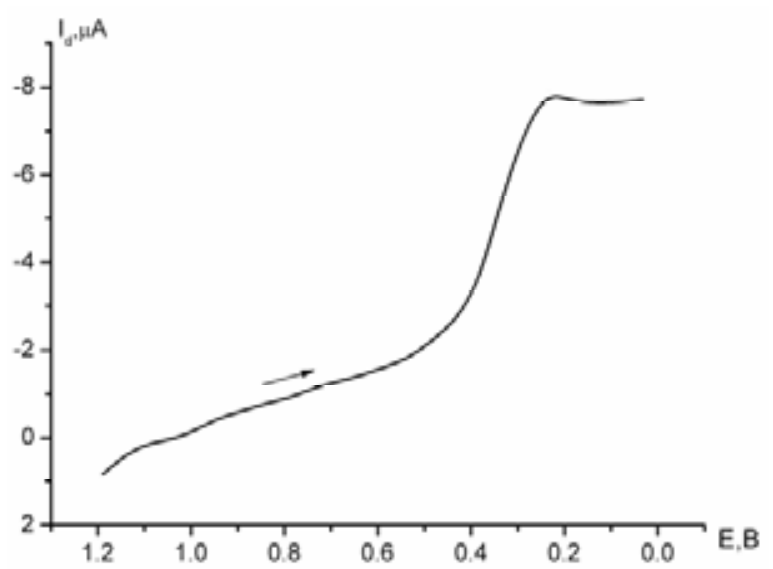

Рис. 1. Циклическая вольтамперограмма модельного раствора ионов церия (III) (Г, $\mathrm{d}_{3}=5$ мм, $0.04 \mathrm{M} \mathrm{HCl}$ $+0.20 \mathrm{M} \mathrm{CH}_{3} \mathrm{COOH}, \mathrm{C}=7.5 \mathrm{мг} /$ л, отн. нас.к.э., $v=25 \mathrm{MB} / \mathrm{c}$ )

Fig.1. Cyclic voltammogram of cerium (III) model solution (G, $\mathrm{d}_{3}=5 \mathrm{~mm}, 0.04 \mathrm{M} \mathrm{HCl}+0.20 \mathrm{M} \mathrm{CH}_{3} \mathrm{COOH}, \mathrm{C}=$ $7.5 \mathrm{mg} \mathrm{l}^{-1}, \mathrm{~V}$ vs SCE, scan rate $25 \mathrm{mV} \mathrm{s}^{-1}$ ) 


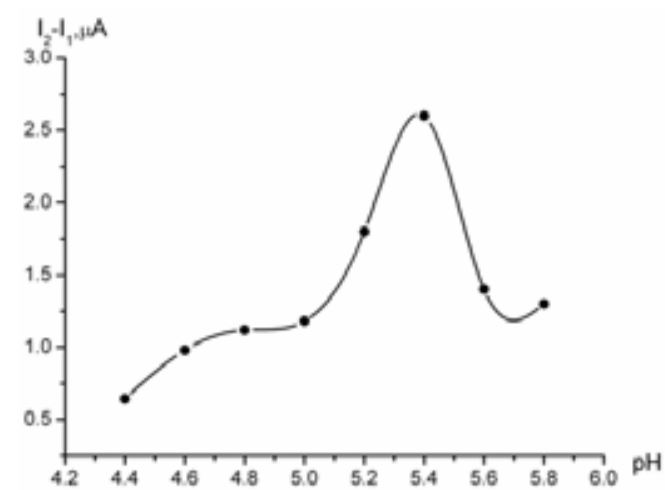

(a)

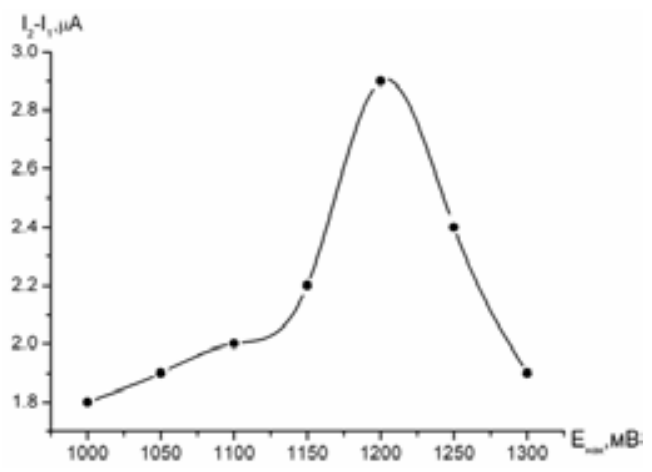

(B)

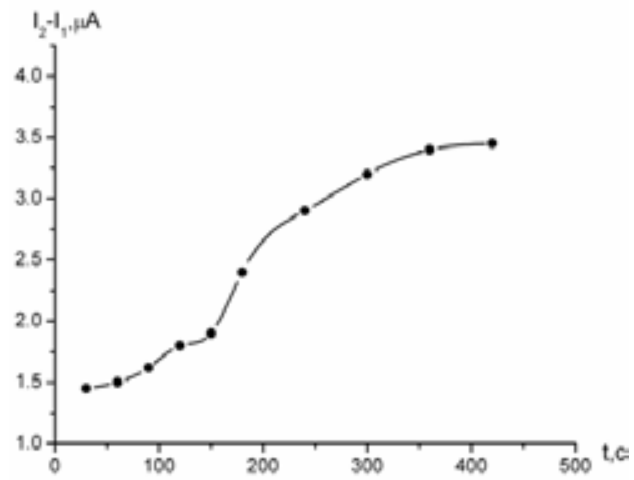

(б)

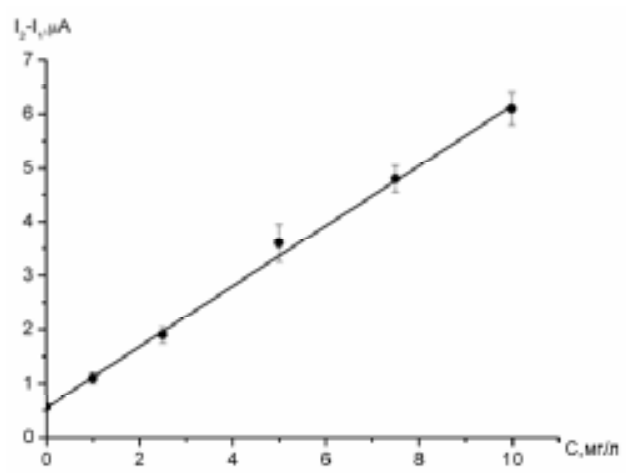

(г)

Рис. 2. Зависимость аналитического сигнала ионов церия (III) (0.5 мг/л) от $\mathrm{pH}$ раствора $\left(\mathrm{t}_{\text {нак }}=420 \mathrm{c}\right.$, $\left.\mathrm{E}_{\text {нак }}=1.2 \mathrm{~B}\right)(\mathbf{a})$; времени накопления $\left(\mathrm{pH} 5.4, \mathrm{E}_{\text {нак }}=1.2 \mathrm{~B}\right)(\boldsymbol{\mathbf { 0 }}) ;$ потенциала накопления $\left(\mathrm{pH} 5.4, \mathrm{t}_{\text {нак }}=420 \mathrm{c}\right)$ (в) $\left(\Gamma, 0.04 \mathrm{M} \mathrm{HCl}+0.20 \mathrm{M} \mathrm{CH}_{3} \mathrm{COOH}\right.$, отн. нас.к.э., v $\left.=25 \mathrm{MB} / \mathrm{c}\right)$. г - Градуировочный график инверсионновольтамперометрического определения ионов церия (III) (Г, $0.04 \mathrm{M} \mathrm{HCl}+0.20 \mathrm{M} \mathrm{CH}_{3} \mathrm{COOH}, \mathrm{pH} 5.4$, $\mathrm{E}_{\text {нак }}=1.2 \mathrm{~B}, \mathrm{t}_{\text {нак }}=420$ с, отн. нас.к.э., $v=25 \mathrm{MB} / \mathrm{c}$ )

Fig.2. Dependence of the analytical signal of cerium (III) $(0.5 \mathrm{mg} / \mathrm{L})$ on solution $\mathrm{pH}\left(\mathrm{t}_{\mathrm{acc}}=420 \mathrm{~s} ; \mathrm{E}_{\mathrm{e}}=1.2 \mathrm{~V}\right)(\mathbf{a})$;

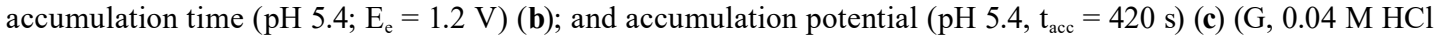
$+0.20 \mathrm{M} \mathrm{CH}_{3} \mathrm{COOH}$; $\mathrm{V}$ vs SCE, scan rate $\left.25 \mathrm{mV} \mathrm{s}^{-1}\right)$. $\mathbf{d}-$ Calibration dependence for determining cerium (III) by stripping voltammetry $\left(\mathrm{G}, 0.04 \mathrm{M} \mathrm{HCl}+0.20 \mathrm{M} \mathrm{CH}_{3} \mathrm{COOH} ; \mathrm{pH} 5.4, \mathrm{E}_{\mathrm{e}}=1.2 \mathrm{~V}, \mathrm{t}_{\mathrm{acc}}=420 \mathrm{~s}, \mathrm{~V}\right.$ vs SCE, scan rate $25 \mathrm{mV} \mathrm{s}^{-1}$ )

Таблица 3. Содержание ионов церия (III) в фильтратах после его сорбции по данным инверсионной вольтамперометрии $(\mathrm{n}=3, \mathrm{p}=0.95)$

Table 3. Concentration of cerium (III) in filtrates after sorption according to stripping voltammetry $(n=3$, $\mathrm{P}=0.95)$.

\begin{tabular}{|c|c|c|c|}
\hline № & Содержание церия, мг/л & Добавка церия, мг/л & Содержание церия с добавкой, мг/л \\
\hline 1 & $43.0 \pm 6.0$ & 50.0 & $94.0 \pm 7.0$ \\
\hline 2 & $36.0 \pm 3.0$ & 40.0 & $74.0 \pm 8.0$ \\
\hline 3 & $14.0 \pm 2.0$ & 20.0 & $33.0 \pm 4.0$ \\
\hline 4 & $12.0 \pm 2.0$ & 15.0 & $26.0 \pm 4.0$ \\
\hline 5 & $7.6 \pm 1.5$ & 10.0 & $17.0 \pm 3.0$ \\
\hline 6 & $3.4 \pm 0.5$ & 5.0 & $9.0 \pm 2.0$ \\
\hline
\end{tabular}




\section{Список литературы}

1. Рябчиков Д.И., Рябухин В.А. Аналитическая химия редкоземельных элементов и иттрия. М.: Наука, 1966. 380 с. [Ryabchikov D.I., Ryabukhin V.A. Analytical chemistry of rare-earth elements and yttrium. M., Nauka, 1966. P. 380. (In Russ.)].

2. Zhang X., Deng Y.-Q., Tian P., Shang H., Xu J., Han Y.-F. Dynamic active sites over binary oxide catalysts: In situ/operando spectroscopic study of low-temperature $\mathrm{CO}$ oxidation over $\mathrm{MnO}_{\mathrm{x}^{-}}$ $\mathrm{CeO}_{2}$ catalysts. Applied Catalysis. B: Environmental 2016. Vol. 191, P. 179-191.

3. Stankovic D.M., Mehmeti E., Zavasnik J., Kalcher K. Determination of nitrite in tap water: A comparative study between cerium, titanium and selenium dioxide doped reduced grapheme oxide modified glassy carbon electrodes. Sensors and Actuators B: Chemical 2016. Vol. 232, P. 311-317.

4. Guo J.-J., Hu A., Chen Y., Sun J., Tang H., Zuo Z. Photocatalytic C-C bond cleavage and amination of cycloalkanols by cerium (III) chloride complex. Angewandte Chemie International Edition 2016. Vol. 55, P. 15319-15322.

5. Luo Y., Calvillo L., Daiguebonne C., Daletou M.K., Granozzi G., Alonso-Vante N. A highly efficient and stable oxygen reduction reaction on $\mathrm{Pt} / \mathrm{CeO}_{\mathrm{x}} / \mathrm{C}$ electrocatalyst obtained via a sacrificial precursor based on a metal-organic framework. Applied Catalysis. B: Environmental 2016. Vol. 189, P. $39-50$.

6. Piña-Pérez Y., Morales F.T., Hernández R.P., Murillo R.A., Acevedo-Pena P., Gomez-Romero R. Photocatalytic activity of $\mathrm{Al}_{2} \mathrm{O}_{3}$ improved by the addition of $\mathrm{Ce}^{3+} \mathrm{Ce}^{4+}$ synthesized by the sol-gel method. Photodegradation of phenolic compounds using UV light. Fuel 2016. Vol. 30, P. 106-117.

7. Löfberg A., Guerrero-Caballero J., Kane T., Rubbens A., Jalowiecki-Duhamel L. Ni/CeO based catalysts as oxygen vectors for the chemical looping dry reforming of methane for syngas production. Applied Catalysis. B: Environmental 2017. Vol. 212, P. 159-174.

8. Kanai S., Nagahara I., Kita Y., Kamata K., Hara M. A bifunctional cerium phosphate catalyst for chemoselective acetalization. Chemical Science 2017. Vol. 8, P. 3146-3153.

9. Turakulova A.O., Kharlanov A.N., Levanov A.V., Isaikina O.Ya, Lunin V.V. Catalytic properties of the $\mathrm{VO}_{\mathrm{x}} / \mathrm{Ce}_{0.46} \mathrm{Zn}_{0.54} \mathrm{O}_{2}$ oxide system in the oxidative dehydrogenation of propane. Russian Journal of Physical Chemistry A 2017. Vol. 91(1), P. 17-25.

10. Mazidi M., Behbahani R.M., Fazeli A. Ce promoted $\mathrm{V}_{2} \mathrm{O}_{5}$ catalyst in oxidation of $\mathrm{SO}_{2}$ reaction. Applied Catalysis B: Environmental 2017. Vol. 17, P. 216-258.

11. Xing M., Sun Q., Zeng C., Wang H., Zhao D., Zhang N., Hong S. Modulating $\mathrm{Cu}^{+}$distribution on the surface of Ce-doped $\mathrm{CuO}$ composite oxides for $\mathrm{SO}_{2}$-resistant $\mathrm{NH}_{3}$-selective catalytic reduction of NO. RCS Advances 2017. Vol. 7, P. 18830-18837.

12. Ali S., Chen L., Yuan F., Li R., Zhang T., Bakhtiar S.H., Leng X., Niu X., Zhu Y Synergistic effect between Copper and Cerium on the performance of $\mathrm{Cu}_{x}-\mathrm{Ce}_{0.5-x}-\mathrm{Zr}_{0.5}(x=0.1-0.5)$ oxides catalysts for selective catalytic reduction of NO with ammonia. Applied Catalysis B: Environmental 2017. Vol. 210, P. 223-234.

13. Shukla K., Srivastava V.C. Diethyl carbonate synthesis by ethanolysis of urea using Ce-Zn oxide catalysts. Fuel Processing Technology 2017. Vol. 161, P. 116-124.

14. Kumar J.V., Karthik R., Chen S.M., Marikkani S., Elangovan A., Muthuraj V. Green synthesis of a novel flower-like cerium vanadate microstructure for electrochemical detection of tryptophan in food and biological samples. Journal of Colloid and Interface Science 2017. Vol. 496, P. 78-86.

$$
-601-
$$


15. Ye T., Huang W., Zeng L., Li M., Shi J. $\mathrm{CeO}_{2-x}$ platelet from monometallic cerium layered double hydroxidesand its photocatalytic reduction of $\mathrm{CO}_{2}$. Applied Catalysis B: Environmental 2017. Vol. 210, P. 141-148.

16. Гайдук О.В., Панталер Р.П., Бланк А.Б. Фотометрическое определение микрограммовых количеств Cе (IV) тропеолином 00. Заводская лаборатория. Диагностика материалов 2006. T. 72(5), C. 12-14 [Gaiduk O.V., Pantaler R.P., Blank A.B. Photometric determination of microgram quantities of Ce (IV) tropeoline 00. Factory laboratory. Diagnosis of materials 2006. T. 72 (5), pp. 12-14 (In Russ.)]

17. Arantes de Carvalho G.G., Kondaveeti S., Petri D.S., Fioroto A.M., Albuquerque L.G., Oliveira P.V. Evaluation of calcium alginate beads for $\mathrm{Ce}, \mathrm{La}$ and $\mathrm{Nd}$ preconcentration from groundwater prior to ICP OES analysis. Talanta 2016. Vol. 161, P. 707-712.

18. Alizadeeh T., Ganjali M.R., Akhoundian M., Norouzi P. Voltammetric determination of ultratrace levels of cerium (III) using a carbon paste electrode modified with nano-sized cerium-imprinted polymer and multiwalled carbon nanotubes. Microchima Acta 2016. Vol. 183(3), P. 1123-1130.

19. Rofouei M.K., Tajarrod N., Masteri-Farahani M., Zadmard R. A new fluorescence sensor for Cerium (III) ion using glycine dithiocarbamate capped manganese doped $\mathrm{ZnS}$ quantum dots. J. Fluorescence 2015. Vol. 25(6), P. 1855-1866.

20. Williams A.N., Phongikaroon S. Elemental detection of Cerium and Gadolinium in aqueous aerosol via laser-induced breakdown spectroscopy. Applied Spectroscopy 2016. Vol. 6, P. 1-9.

21. Bulgakov R.G., Gazeeva D.R., Galimov D.I. Chemiluminescence of the $\mathrm{Ce}^{3+}$ ions, and the ${ }^{1} \mathrm{O}_{2}$ and $\left({ }^{1} \mathrm{O}_{2}\right)_{2}$ molecular species of oxygen induced by active surface of the $\left(\mathrm{NH}_{4}\right)_{2} \mathrm{Ce}\left(\mathrm{NO}_{3}\right)_{6}$ crystals at reduction of $\mathrm{Ce}^{4+}$ to $\mathrm{Ce}^{3+}$ by water in heterogeneous system « $\left(\mathrm{NH}_{4}\right)_{2} \mathrm{Ce}\left(\mathrm{NO}_{3}\right)_{6}-\mathrm{C}_{6} \mathrm{H}_{6}-\mathrm{H}_{2} \mathrm{O} »$. J. Luminescence 2016. Vol. 183, P. 159-165.

22. Yang X., Hao Z., Shen M., Yi R., Li J., Yu H., Guo L., Li X., Zeng X., Lu Y. Simultaneous determination of $\mathrm{La}, \mathrm{Ce}, \mathrm{Pr}$, and $\mathrm{Nd}$ elements in aqueous solution using surface-enhanced laser-induced breakdown spectroscopy. Talanta 2016. Vol. 163, P. 127-131.

23. Kuchekar S.R., Naval R.M., Han S. Solvent extraction and spectrophotometric determination of Cerium(IV) by using $o$-methoxy phenylthiourea as an analytical reagent. Solvent Extraction research and development, Japan 2016. Vol. 23(1), P. 19-29.

24. Rodionova O.Ye., Pomerantsev A.L. Non-linear multivariate curve resolution applied to the spectrophotometric determination of cerium (III) in aqueous nitric acid solutions for process control. Analytical methods 2016. Vol. 8(2), P. 435-444.

25. Srivastava B., Barman M.K., Chatterjee M., Roy D., Mandal B. Solid phase extraction, separation and preconcentration of rare elements thorium(IV), uranium(VI), zirconium(IV), cerium(IV) and chromium (III) amid several other foreign ions with eriochrome black T anchored to 3-D networking silica gel. J. Chromatography A 2016. Vol. 1451, P. 1-14.

26. Zinin D.S., Bushuev N.N., Kuznetsov V.V. X-ray fluorescence determination of La, Ce, Pr, $\mathrm{Nd}$, and $\mathrm{Sm}$ in industrial sediments of calcium sulfate using linear regression analysis. J. Analytical Chemistry 2017, Vol. 72(3), P. 279-288.

27. Suvorova D., Khudonogova E., Revenko A. X-ray fluorescence determination of Cs, Ba, La, $\mathrm{Ce}, \mathrm{Nd}$, and Ta concentrations in rocks of various composition. X-Ray Spectrometry 2017. Vol. 46(3), P. 200-208. 
28. Будников Г.К., Майстренко В.Н., Вяселев М.Р. Основы современного электрохимического анализа. М., Мир, 2003. 592 с. [Budnikov G.K., Maistrenko V.N., Vyaselev M. R. Fundamentals of modern electrochemical analysis. M., Mir, 2003. 592 p. (In Russ.)]

29. Матакова, Р.Н., Сагадиева К.Ж. Электрохимия редкоземельных металлов. Вестник КазНУ. Серия химич. 2012. Т. 65(2), C. 114 - 124. [Matakova R.N., Sagadiyeva K.Zh. Electrochemistry of rare-earth metals. Chemical bulletin of Kazakh National University 2012. Vol. 65(2), P. 114-124. (In Russ.)]

30. Матакова, Р.Н. Проблемы электрохимического анализа следовых содержаний крайне электроотрицательных металлов. Вестник КазНУ. Серия химич. 2013. Т. 69(1), С. 6-10. [Matakova R.N. Problems of electrochemical analysis of trace contents of extremely electronegative metals. Chemical bulletin of Kazakh National University 2013. Vol. 69(1), P. 6-10. (In Russ.)]

31. Бадавамова, Г.Л. Исследование процессов разряда-ионизации церия на стеклоуглеродном электроде в минерально-органических средах. Вестник КазНУ. Серия химич. 2003. Т.29(1), C. 16 - 20. [Badavamova G.L. Study of the processes of discharge-ionization of cerium on a glassy carbon electrode in mineral-organic environment. Chemical bulletin of Kazakh National University 2003. Vol. 29(1), P. 16-20. (In Russ.)]

32. Матакова Р.Н., Бадавамова Г.Л. Следовый электрохимический анализ европия, иттербия и церия при совместном присутствии в растворе. Вестник КазНУ. Серия химич. 2012. T. 65(1), C. 127-132. [Matakova R.N., Badavamova G.L. Trace electrochemical analysis of europium, ytterbium, and cerium under their joint presence in solution. Chemical bulletin of Kazakh National University 2012. Vol. 65(1), P. 127-132. (In Russ.)].

33. Матакова, Р.Н., Бадавамова Г.Л. Электрохимические особенности поведения и анализа низких содержаний редкоземельных элементов на жидких и твердых электродах. Вестник КазНУ. Серия химич. 2003. T.31(3), C. 223-225. [Matakova R.N., Badavamova G.L. Electrochemical characteristics of behavior and analysis of low contents of rare-earth elements on liquid and solid electrodes. Chemical bulletin of Kazakh National Universiry 2003. Vol. 31(3), P. 223-225. (In Russ.)].

34. Matakova, R.N. Study of the discharge ionization of cerium at a solid-paste graphite electrode. Journal of Analytical Chemistry 1999. Vol. 54(11), P. 1053-1055.

35. Javanbakht, M. Stripping voltammetry of cerium (III) with a chemically modified carbon paste electrode containing functionalized nanoporous silica gel. Electroanalisis 2005. Vol. 20(2), P. 203-206.

36. Представление результатов химического анализа (рекомендации IUPAC 1994 г.) Пер. с англ. Проскурнина М.А. Журн. аналит. химии. 1998. Т. 53(9), С. 999-1008. [Predstavlenie rezul'tatov khimicheskogo analiza (rekomendatsii IUPAC 1994 g.) Presentation of the Results of Chemical Analysis (IUPAC Recommendations 1994). Zh. anal. khim. 1998. Vol. 53(9), P. 999-1008. (In Russ.)]. 